\title{
ON NETWORK FLOW EQUATIONS AND SPLITTING FORMULAS FOR SOJOURN TIMES IN QUEUEING NETWORKS ${ }^{1}$
}

\author{
HANS DADUNA \\ Institut für Mathematische Stochastik \\ Universität Hamburg \\ Bundesstraße 55, D-2000 Hamburg 19, GERMANY
}

\begin{abstract}
LEMOINE's network flow equations are generalized to the case of multiserver networks. These equations provide a basis for recursive evaluation of residual conditional sojourn time moments.

Key words: $\quad$ Multiserver queues, Jackson networks, sojourn time distributions, moment formulas, flow equations.
\end{abstract}

AMS (MOS) subject classification: $\quad 60 \mathrm{~K} 25,90 \mathrm{~B} 22,68 \mathrm{C} 15$.

\section{INTRODUCTION}

Recently, LEMOINE [3] derived for JACKSON networks of single server nodes a set of "network flow equations" which as he claimed are a first step towards obtaining higher moments of sojourn times for customers in networks of queues. These equations connect a customer's residual sojourn time distributions given the node he has just entered in his passage through the network. Nothing is said in this expressions about the customer's itinerary through the network.

It is the purpose of this paper to sketch how these network flow equations can be obtained from "splitting formulas" for passage time distributions which are previously obtained, at the same time generalizing LEMOINE's result to the case of JACKSON networks with multiserver nodes.

\section{THE NETWORK FLOW EQUATIONS}

We consider a JACKSON network of nodes $\{1, \ldots, J\}=\widetilde{J}$, node $i$ being a multiserver with $m_{i} \geq 1$ service channels and infinite waiting room under first-come-first-served (FCFS) discipline.

At node $i \in \widetilde{J}$, customers arrive in a Poisson $\left(\lambda_{i}\right)$ stream, $\lambda_{i} \geq 0$, and service times are exponentially distributed with mean $\mu_{i}{ }^{-1}$.

\footnotetext{
${ }^{1}$ Received: April 1990, Revised: January 1991
} 
Upon leaving node $i$, a customer either jumps to node $j$ with probability $r_{i j} \geq 0$ or leaves the network with probability $r_{i 0} \geq 0$. We assume the routing to be Markovian and the family of all service and interarrival times to be independent and independent from routing.

Let $r_{0 i}=\left\{\begin{array}{cc}1 & i=0 \\ 0 & i=1, \ldots, J\end{array}\right.$, and $R=\left(r_{i j}: i, j=0, \ldots, J\right)$. We assume that the stochastic matrix $R$ defines a finite state Markov chain $Y=(Y(n): n \in N)$, which is absorbing at 0 for any initial state in finite time with probability 1.

The network's behavior over time can be described by a strong Markov process $X=\left(X(t): t \geq 0\right.$ with state space $N^{J}$, describing the joint queue lengths at the nodes including customers in service.

The coordinate processes of $X$ are denoted by $\left(X_{i}(t): t \geq 0\right)=X_{i}, i=1, \ldots, J$.

We assume $X$ to be ergodic, which is guaranteed by the condition

$$
\alpha_{i}<m_{i} \mu_{i}, \quad i=1, \ldots, J
$$

(where $\left(\alpha_{1}, \ldots, \alpha_{J}\right)=\alpha$ is the unique solution of the traffic equation

$$
\alpha=\left(\lambda_{1}, \ldots, \lambda_{J}\right)+\alpha \cdot R
$$

together with the requirement that all nodes are visited by customers. The unique steady state distribution $\pi$ of $X$ is given as follows:

Let $\quad a_{i}(k)=\left\{\begin{array}{ll}k \mu_{i} & k<m_{i} \\ m_{i} \mu_{i} & k \geq m_{i}\end{array} \quad, \quad i=1, \ldots, J\right.$.

then

$$
\pi\left(n_{1}, \ldots, n_{J}\right)=\prod_{i=1}^{J}\left(\prod_{k_{i}=1}^{n_{i}} \frac{\alpha_{i}}{a_{i}\left(k_{i}\right)}\right) \cdot G^{-1},\left(n_{1}, \ldots, n_{J}\right) \in \mathrm{N}^{J}
$$

where $G$ is the norming constant.

According to the celebrated theorem on arrival and departure time distribution (SEVCIK/MITRANI [6] and LAVENBERG/REISER [1]), $\pi$ is the distribution of the system seen by an arriving or jumping customer in equilibrium if he himself is not counted.

For $i \in\{1, \ldots, J\}$ let $\tau_{i}$ be an arrival epoch of a test customer $C$ in equilibrium at node $i$, $\tau_{i}+T_{i}$ the epoch of departure from the network for $C$, i.e., $T_{i}$ is the total remaining time in the network for the customer arriving at $\tau_{i}$ at node $i$, where we do not distinguish between internal (departing from some node, jumping to $i$ ) and external arrivals. 
For the case of single-server node networks, LEMOINE [3] derived a set of "network flow equations" for the Laplace-Stieltjes transforms (LST)

$$
h_{i}(\Theta)=E\left[e^{-\Theta T_{i}}\right], \operatorname{Re}(\Theta) \geq 0, i=1, \ldots, J,
$$

which, as he proved, are equivalent to a set of equations, better suited for the evaluation of moments. We derive a similar set of "network flow equations" for multiserver queueing networks. We state it here as:

Theorem. Let $p_{i}(w)$ denote the steady-state probability that at least $m_{i}$ customers are present at node $i, i=1, \ldots, J$.

Then for $i=1, \ldots, J$ and $\operatorname{Re}(\Theta) \geq 0$

$$
\begin{gathered}
E\left[e^{-\Theta T_{i}}\right]= \\
=r_{i 0} \frac{\mu_{i}}{\mu_{i}+\Theta} \cdot\left\{1-p_{i}(w)\left(1-\frac{m_{i} \mu_{i}-\alpha_{i}}{m_{i} \mu_{i}-\alpha_{i}+\Theta}\right)\right\}+ \\
+\sum_{j=1}^{J} r_{i j} E\left[\frac{\mu_{i}}{\mu_{i}+\Theta} \cdot\left(\frac{m_{i} \mu_{i}}{m_{i} \mu_{i}+\Theta}\right)^{\left(X_{i}\left(\tau_{j}-\right)+1-m_{i}\right)+} \cdot e^{-\Theta T_{j}}\right]
\end{gathered}
$$

with the convention that the test customer $C$ is not counted in the population vector

$$
X(t)=\left(X_{1}(t), \ldots, X_{J}(t)\right), t \geq 0
$$

Remark. (1) For the case $m_{i}=1, i=1, \ldots, J$ this is formula (52) of LEMOINE [3].

(2) To obtain residual sojourn time moments the expressions of the theorem should be differentiated and evaluated at $\Theta=0$. But then the same problems arise as in LEMOINE's [3] procedure: There are too many unknowns in the set of equations obtained. Therefore further equations have to be derived which are independent from the above. For a discussion and an example see LEMOINE [3].

Proof. For the test customer $C$, we set a condition to the distribution of his residual sojourn time in the network on the sequence of nodes he will visit before eventually leaving the network, i.e., on the possible behavior of $Y=(Y(n): n \in N)$ given $P(Y(0)=i)=1$, and governed by the matrix $R$ which describes $C$ 's future journey through the network until absorption. Let $G$ be the state space describing the possible behavior of $Y$, i.e.,

$$
g \in G \Leftrightarrow g(g(0), g(1), \ldots) \in\{0,1, \ldots, J\}^{\mathbf{N}}
$$


with $g(n)=0 \Rightarrow g(n+k)=0 \forall k \in \mathbb{N}$

and $g(n) \neq 0$ for only a finite number of steps.

We denote by

$$
G_{j}=\{g \in G: g(0)=j\}, j=1, \ldots, J
$$

Then $h_{i}(\Theta)=E\left[e^{-\Theta T_{i}}\right]=$

$$
=\sum_{g \in G_{i}} \operatorname{Pr}(Y=g \mid Y(0)=i) E\left[e^{-\Theta T_{i}} \mid Y=g\right]
$$

and $E\left[e^{-\Theta T_{i}} \mid Y=g\right]$ is the LST of the passage time distribution for customer $C$ traversing the prescribed path $\widetilde{g}=(g(0)=i, g(1), \ldots, g(\widetilde{n}))$ through a network with Markovian routing, where $\widetilde{n}=\widetilde{n}(g)=\max (k: n(k) \neq 0)$. So $T_{i}=\sum_{k=0}^{\tilde{n}} V_{g(k)}$, where $V_{g(k)}$ is $C$ 's sojourn time at node $g(k)$.

We now transform the network into a network with deterministic routing (see MELAMED [4]) by introducing different customer types:

A customer entering the network at node $i$ is of type $g \in G_{i}$ with probability

$$
\operatorname{Pr}(Y=g \mid Y(0)=i)
$$

and has itinerary $\widetilde{g}$ during his visit at the network.

Having obtained the network with deterministic routing, Lemma 2.1 and Theorem 2 in SCHASSBERGER/DADUNA [5] can be applied. This provides ( $i$ ) the joint distribution of $C$ 's sojourn time at node $i$ and the state of the network at $C$ 's departure from node $i$ proceeding to node $g(1)$, and (ii) a "splitting formula" which represents the total passage time through a path as a sum of the sojourn time in $i$ and the residual passage time through $(g(1), g(2), \ldots, g(\tilde{n}))$ both conditioned on the state of network in $C$ 's departure epoch from node $i$.

Turning back to the network with random routing, i.e., deconditioning over all customer types (but leaving $C$ 's itinerary fixed) we obtain

$$
\begin{aligned}
& E\left[e^{-\Theta T_{i}} \mid Y=g\right]= \\
& =\sum_{\left(n_{1}, \ldots, n^{J}\right) \in \mathbb{N}^{J}} \pi\left(n_{1}, \ldots, n_{J}\right) \cdot \frac{\mu_{i}}{\mu_{i}+\Theta} \cdot\left(\frac{m_{i} \mu_{i}}{m_{i} \mu_{i}+\Theta}\right)^{\left(n_{i}+1-m_{i}\right)}+.
\end{aligned}
$$




$$
\cdot E\left[e^{-\Theta\left(V_{g(1)}+V_{g(2)}+\ldots+V_{g(\tilde{n})}\right)} \mid Y=g, X\left(\tau_{i}+V_{i}\right)=\left(n_{1}, \ldots, n_{g(1)}+1, \ldots, n_{J}\right)\right]
$$

where $a_{+}=\max (0, a)$ for $a \in \mathbb{R}$, and $C$ is included in $X\left(\tau_{i}+V_{i}\right)$.

Now $(g(1), \ldots, g(\tilde{n}), 0,0, \ldots) \in G_{g(1)}$, and from the strong Markov property of $X$ it follows:

$$
\begin{aligned}
& h_{i}(\Theta)= \\
& =\sum_{g \in G_{i}} r_{i, g(1)} \cdot \operatorname{Pr}(Y=(g(1), g(2), \ldots, g(\widetilde{n}), 0,0, \ldots,) \mid Y(0)=g(1)) \cdot \\
& \cdot \sum_{\left(n_{1}, \ldots, n_{J}\right) \in N^{J}} \pi\left(n_{1}, \ldots, n_{J}\right) \frac{\mu_{i}}{\mu_{i}+\Theta}\left(\frac{m_{i} \mu_{i}}{m_{i} \mu_{i}+\Theta}\right)^{\left(n_{i}+1-m_{i}\right)}+. \\
& \cdot E\left[e^{-\Theta\left(V_{g(1)}+\ldots+V_{g(\tilde{n})}\right)} \mid Y=g, X\left(\tau_{i}+V_{i}\right)=\left(n_{1}, \ldots, n_{g(1)}+1, \ldots, n_{J}\right)\right]= \\
& =\sum_{j=0}^{J} r_{i j} \sum_{\left(n_{1}, \ldots, n_{J}\right) \in N^{J}} \pi\left(n_{1}, \ldots, n_{J}\right) \frac{\mu_{i}}{\mu_{i}+\Theta} \cdot\left(\frac{m_{i} \mu_{i}}{m_{i} \mu_{i}+\Theta}\right)^{\left(n_{i}+1-m_{i}\right)}+. \\
& \sum_{\substack{g^{\prime} \in G_{j} \\
(g(1), \ldots, g(\tilde{n}), 0,0, \ldots) .}} P\left(Y=g^{\prime} \mid Y(0)=j\right) . \\
& g^{\prime}=(g(1), \ldots, g(\tilde{n}), 0,0, \ldots) \text {. } \\
& \cdot E\left[e^{-G\left(V_{g(1)}+\ldots+V_{g(\tilde{n})}\right)} \mid(Y(k)=g(k), k \geq 1), X\left(\tau_{i}+V_{i}\right)=\left(n_{1}, \ldots, n_{g(1)}+1, \ldots, n_{J}\right)\right] \\
& =\sum_{j=0}^{J} r_{i j} \sum_{\left(n_{1}, \ldots, n_{J}\right) \in N^{J}} \pi\left(n_{1}, \ldots, n_{J}\right) \frac{\mu_{i}}{\mu_{i}+\Theta} \cdot\left(\frac{m_{i} \mu_{i}}{m_{i} \mu_{i}+\Theta}\right)^{\left(n_{i}+1-m_{i}\right)}+. \\
& \cdot E\left[e^{-\Theta T} j \mid X\left(\tau_{j}\right)=\left(n_{1}, \ldots, n_{j}+1, \ldots, n_{J}\right)\right]
\end{aligned}
$$

Remarks.(3) In an analogous manner as we obtained here from the "splitting formula" of SCHASSBERGER/DADUNA [5] the "network flow equation" for networks with multiserver nodes, and a single customer class, we can deal with random routing networks with different customer types.

The case of a single server network with different customer types can be derived from DADUNA [1], where the splitting formula for a customer's passage time through a fixed path in a 
network with random routing appears as equation (7) which leads directly to our Theorem or the analog to LEMOINE's [3] formula (52). For this case, LEMOINE's first version of the "network flow equation" (see (6) in LEMOINE [3]) follows directly from formula (5) in DADUNA [1].

(4) Network flow equations for mixed networks can be derived in exactly the same way providing again formulas for recursive evaluation of residual conditional sojourn time moments for external customers. In fact, the splitting formula in SCHASSBERGER/DADUNA [5] is proved for that case.

\section{REFERENCES}

[1] Daduna, H., "On passage times in JACKSON networks: Two-stations walk and overtakefree paths", Zeitschrift f. Oper. Res. 27, ser. A., (1983), 239-256.

[2] Lavenberg, S.S. and Reiser, M., "Stationary state probabilities at arrival instants for closed queueing networks with multiple types of customers", J. Appl. Prob. 17, (1980), 1048-1061.

[3] Lemoine, A.J., "On sojourn time in JACKSON networks of queues", J. Appl. Prob. 24, (1987), 495-510.

[4] Melamed, B., "Sojourn times in queueing networks", Math. Oper. Res. 7, (1982), 223-244.

[5] Schassberger, R. and Daduna, H., "Sojourn times in queueing networks with multiserver nodes", J. Appl. Prob. 24, (1987), 511-521.

[6] Sevcik, K.C., and Mitrani, I., "The distribution of queueing network states at input and output instants", J. Ass. Comp. Mach. 28, (1981), 358-371. 


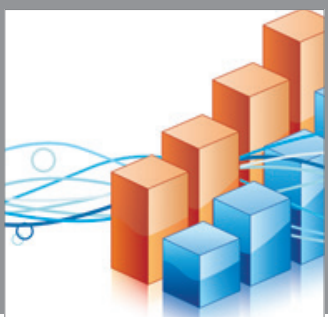

Advances in

Operations Research

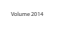

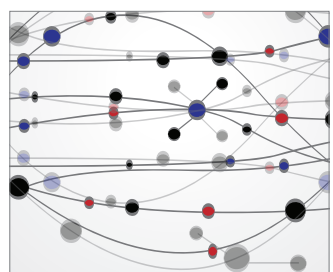

\section{The Scientific} World Journal
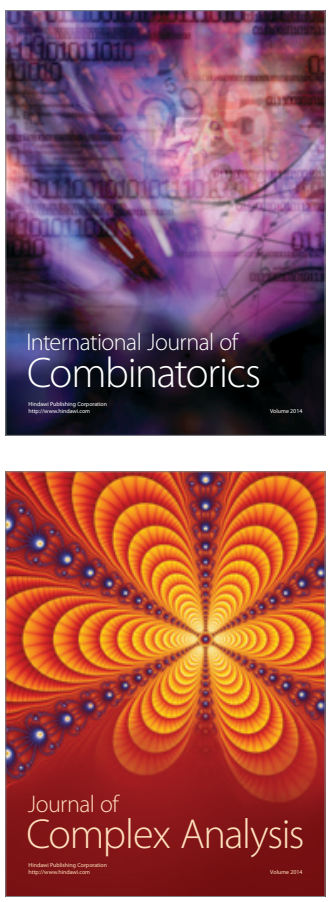

International Journal of

Mathematics and

Mathematical

Sciences
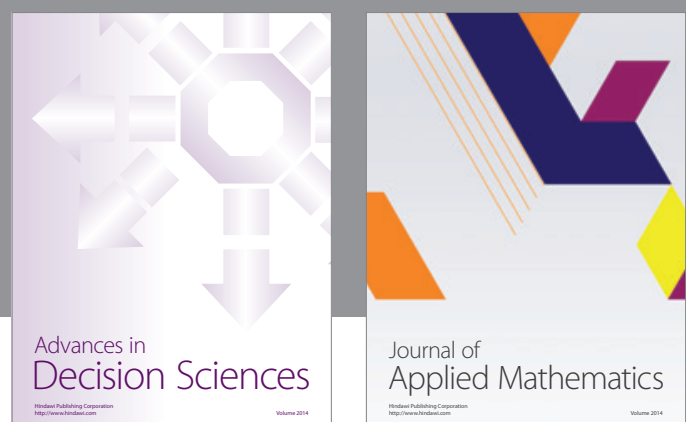

Journal of

Applied Mathematics
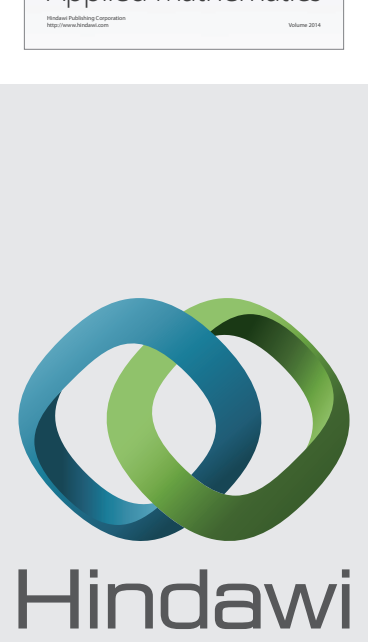

Submit your manuscripts at http://www.hindawi.com
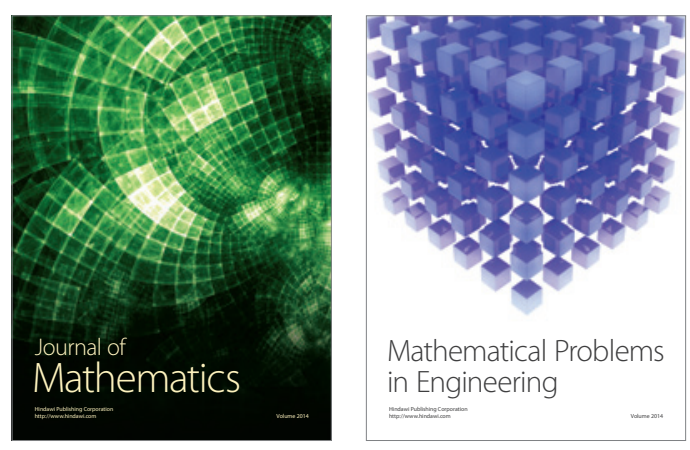

Mathematical Problems in Engineering
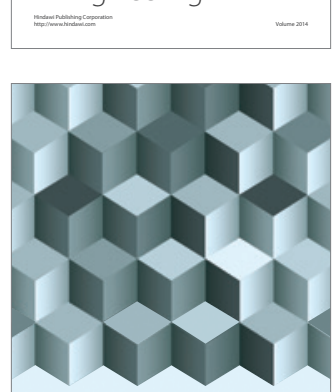

Journal of

Function Spaces
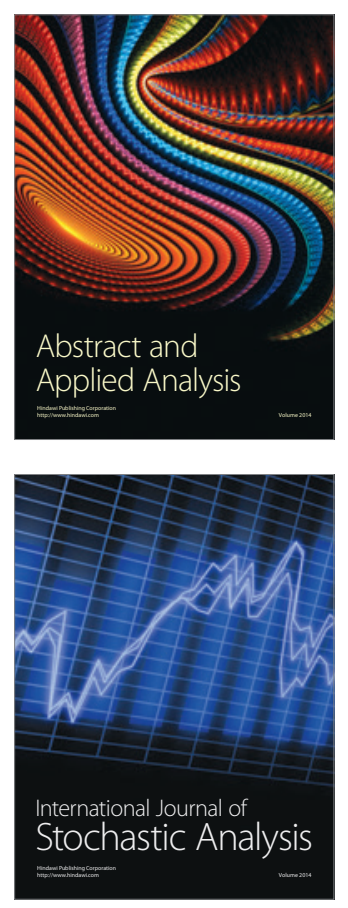

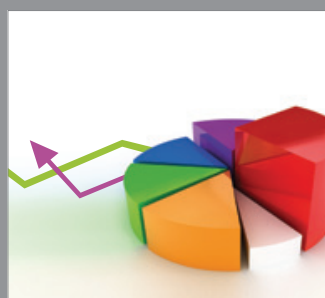

ournal of

Probability and Statistics

Promensencen
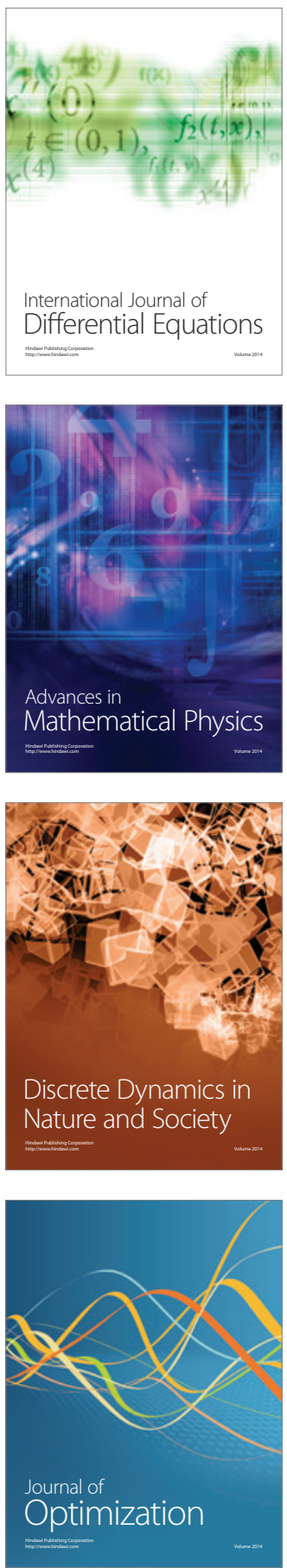